\title{
Investigations of heat transfer of copper-in-Therminol 59 nanofluids
}

Wenhua Yu $^{\mathrm{a}, *}$, Elena V. Timofeeva ${ }^{\mathrm{a}}$, Dileep Singh ${ }^{\mathrm{b}}$, David M. France ${ }^{\mathrm{a}, \mathrm{c}}$, Roger K. Smith ${ }^{\mathrm{a}}$

${ }^{\text {a} E n e r g y ~ S y s t e m s ~ D i v i s i o n, ~ A r g o n n e ~ N a t i o n a l ~ L a b o r a t o r y, ~} 9700$ South Cass Avenue, Argonne, IL 60439, USA

buclear Engineering Division, Argonne National Laboratory, 9700 South Cass Avenue, Argonne, IL 60439, USA

${ }^{c}$ Department of Mechanical and Industrial Engineering, University of Illinois at Chicago, 842 West Taylor Street (m/c 251), Chicago, IL 60607, USA

\begin{abstract}
Convective turbulent-flow heat transfer experiments were performed with Therminol 59-based nanofluids containing copper nanoparticles at particle volume concentrations of $0.50 \%$ and $0.75 \%$. These nanofluids have the unusual properties of being significantly above the thermal conductivity predictions of the effective medium theory with high dynamic viscosities. The friction factors and heat transfer coefficients of the nanofluids were experimentally determined and compared to the predictions from the standard correlation equations. The experimental heat transfer coefficient enhancements were also compared to the predicted heat transfer coefficient ratios of the nanofluids over the base fluid using their thermophysical properties. Finally, based on the measured thermophysical properties and heat transfer coefficients of the nanofluids, the
\end{abstract}

\footnotetext{
${ }^{*}$ Corresponding author. Tel.: +1 630252 7361; fax: +1 6302525568 .

E-mail addresses: wyu@anl.gov (W. Yu), etimofeeva@anl.gov (E.V. Timofeeva), dsingh@anl.gov (D. Singh), dfrance@uic.edu (D.M. France), rk.smith@anl.gov (R.K. Smith).
} 
effect of elevated temperature on the heat transfer coefficient ratios of the nanofluids over the base fluid were evaluated.

Keywords: nanofluid; convective heat transfer; turbulent flow; heat transfer prediction; heat transfer enhancement

\section{Nomenclature}

$c_{p} \quad$ mass-specific heat capacity $(\mathrm{J} / \mathrm{kgK})$

$d_{i} \quad$ inside diameter $(\mathrm{m})$

$d_{o} \quad$ outside diameter $(\mathrm{m})$

E voltage drop (V)

$f \quad$ friction factor

$h \quad$ heat transfer coefficient $\left(\mathrm{W} / \mathrm{m}^{2} \mathrm{~K}\right)$

I current (A)

$k \quad$ thermal conductivity $(\mathrm{W} / \mathrm{mK})$

$m \quad$ mass $(\mathrm{kg})$

Mo Mouromtseff number $\left(\mathrm{J} / \mathrm{m}^{2.6} \mathrm{~s}^{0.2} \mathrm{~K}\right)$

Pr Prandtl number

$q \quad$ heat $(\mathrm{J})$

$\dot{q} \quad$ heat flow rate (W)

$\dot{q}^{\prime} \quad$ heat flow rate per unit length $(\mathrm{W} / \mathrm{m})$

$\dot{q}^{\prime \prime} \quad$ heat flux $\left(\mathrm{W} / \mathrm{m}^{2}\right)$ 


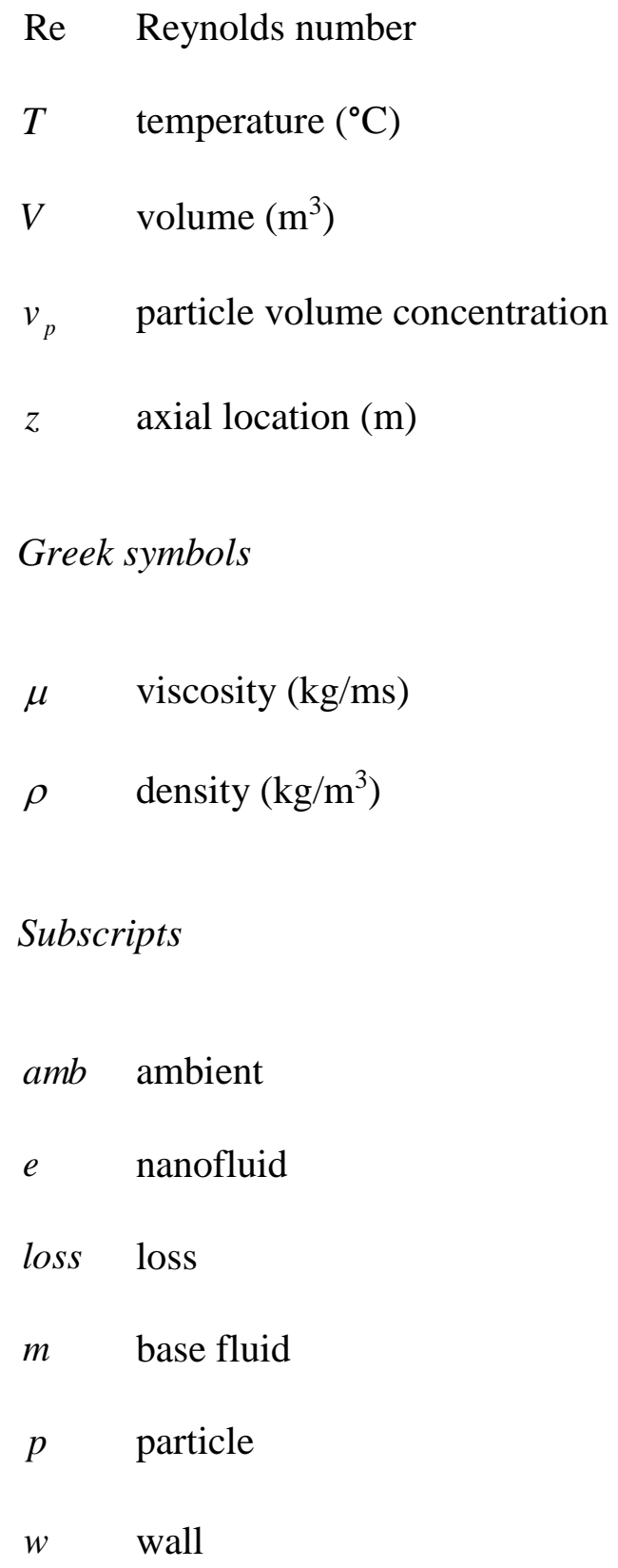

\section{Introduction}

Therminol 59 is a synthetic heat transfer fluid that remains a stable liquid within its operating temperature range of $-45^{\circ} \mathrm{C}$ to $315^{\circ} \mathrm{C}$. It is used in a wide variety of industries such as oil and gas processing, pharmaceuticals manufacturing, and chemical production. However, the heat 
transfer performance of Therminol 59 is generally poor due mainly to its low thermal conductivity and high dynamic viscosity. Therefore, there are challenges and opportunities to improve the heat transfer performance of Therminol 59 by developing Therminol 59-based nanofluids.

Nanofluids are nanotechnology-based heat transfer fluids that are engineered by stably dispersing nanometer-sized solid particles in conventional heat transfer fluids at low particle volume concentrations. From the viewpoint of heat transfer, one of the key benefits of nanofluids is their enhanced effective thermal conductivities compared to those of their base fluids. As the most-studied topic, the effective thermal conductivity enhancements of nanofluids with dispersions of various particles such as ceramics, metals, alloys, semiconductors, nanotubes, and composite particles have been widely investigated since the origination of the nanofluid concept more than a decade ago [1-7]. For nanofluids containing spherical or near-spherical particles, while their effective thermal conductivities are generally predicted approximately by the effective medium theory such as the Maxwell equation [8] and the Bruggeman equation [9], many exceptions exist. This phenomenon is especially observable for nanofluids with metal particle suspensions where their effective thermal conductivities usually are higher (often much higher) than the predictions of the effective medium theory.

Another critical consequence of dispersing particles in a base fluid is the increase of its effective dynamic viscosity. Unfortunately, the increased dynamic viscosity has negative influence on the heat transfer performance of the nanofluid. The investigations on the effective dynamic viscosities of nanofluids are not as comprehensive as those on the effective thermal conductivities of nanofluids. While controversies exist, several general trends for spherical or near-spherical particle suspensions can still be deduced from the engineering literature $[2,4,10]$ 
including (a) that the effective dynamic viscosity of a nanofluid is usually higher than the prediction of the classical Einstein equation [11], (b) that the dynamic viscosity ratio of a nanofluid over its base fluid increases with nanofluid particle volume concentration increase, (c) that the dynamic viscosity ratio of a nanofluid over its base fluid often decreases as the nanofluid temperature increases for a particular particle volume concentration, and (d) that other nanofluid characteristics such as the base fluid dynamic viscosity, the particle size, the particle distribution, and the particle agglomeration also play very important roles in the dynamic viscosity increase of a nanofluid.

For turbulent-flow nanofluids that follow the effective medium theory for thermal conductivity enhancements and that have moderate viscosities, it was seen [12] that the heat transfer coefficient enhancements are predictable from standard liquid correlation equations using the thermophysical properties of the nanofluids. In this paper we investigate whether the heat transfer coefficient can be estimated from thermophysical properties for nanofluids whose effective thermal conductivities don't follow the effective medium theory predictions. Suspensions of metallic copper nanoparticles in Therminol 59 were used for this case study. From a heat transfer perspective, using Therminol 59 as a base fluid of a nanofluid introduces not only the detrimental condition of a high viscosity generally outside the range of most other nanofluid studies but also the thermal conductivity decreasing with the temperature increase. In addition, using copper particles produces the positive condition of a very high thermal conductivity enhancement over the base fluid well above the predictions of the effective medium theory where most of the previous studies have focused. Because of these rather unique properties of copper in Therminol 59 nanofluids, it is important to investigate their heat transfer behavior and correlation to standard fluid equations. Accordingly, the main purpose of the 
present study is to experimentally and theoretically investigate the heat transfer performance of Therminol 59-based nanofluids containing copper nanoparticles at low volume concentrations. Specifically, the present study focuses on (a) experimentally measuring the nanofluid heat transfer coefficients, (b) theoretically analyzing the relationship of the nanofluid heat transfer coefficients and the nanofluid thermophysical properties, (c) comparing the experimental nanofluid heat transfer coefficient enhancements with the theoretical predictions based on the nanofluid thermophysical properties, and (d) evaluating the temperature effects on the nanofluid heat transfer performance.

\section{Nanofluid syntheses}

In the present study, commercially available Therminol 59 and Therminol 66 (Solutia, Inc.) were initially considered as the base fluid for the investigation of nanofluid heat transfer performance. Both fluids have similar thermophysical properties at high temperatures. However Therminol 59 was selected for its lower viscosity at the ambient temperature, due to the pumping restrictions.

The copper nanoparticles used in the present study were synthesized by the chemical reduction of $\mathrm{CuSO}_{4}$ with $\mathrm{NaPO}_{2} \mathrm{H}_{2}$ [13]. Imaging with electron microscopy shows the average particle size of as-synthesized copper nanoparticles being 50-100 nm. The synthesized nanoparticles are phase pure copper, as evidenced by the x-ray diffraction.

The formation of a stable dispersion of the synthesized copper nanoparticles in the base fluid Therminol 59 is a key step to produce practical nanofluids. Therminol 59 is a non-polar fluid comprised of alkildiphenyl mixtures. Thus the best way to achieve stable suspensions is to reduce particle sizes and to reach steric stabilization using surfactants with their molecules 
covering the nanoparticle surfaces and therefore preventing nanoparticles from agglomeration. We used digestive ripening technique [14] to achieve homogenous particle sizes. A combination of two surfactants, benzalkonium chloride and octadecyl thiol, was used for this process and further suspension stabilization. These surfactants were added at the same volumetric proportion as the copper particle volume concentration in the final fluids. Ripening process was conducted by combination of sonication and temperature $\left(140{ }^{\circ} \mathrm{C}\right)$ treatment over 12 hours. The resulting suspensions show high stability and thermal conductivity values and low viscosity increases.

\section{Experimental apparatus}

As shown in the schematic diagram of the heat transfer experimental apparatus in Fig. 1, the test fluid was pumped through the test loop by a turbine pump (MTH Pumps, Model T41CAB) and the test system was open to the atmosphere through the fill port at the inlet of the flowmeter. The flowrate through the experimental test section was adjusted with the by-pass flow control valve. Exiting the pump, the test fluid flowed through the flowmeter (Omega Engineering, Inc., Model FLMH-1201SS-MA), in which volumetric flowrates $Q$ were measured. A thermocouple probe $T_{F M}$ (Omega Engineering, Inc.) just upstream from the flowmeter provided a means to determine the density of the test fluid and subsequently the mass flowrate of the test fluid. The test fluid then entered the preheater where, for a given test, the test fluid temperature was raised to the desired temperature level, monitored through the in-stream thermocouple $T_{i n^{\prime}}$ located at the outlet of the preheater. The preheater, consisting of an AISI type 304 stainless steel tube with a 4.572- $\mathrm{mm}$ inside diameter, a 6.096- $\mathrm{mm}$ outside diameter, and a 500-mm resistance-heated length, was heated by passing current through its wall to generate resistance heat. A direct 
current power supply (Sorensen Company, Model DCR 16-625T) was used, the output power of which could be regulated from 0 to $10 \mathrm{~kW}$ with the maximum voltage drop and the maximum current being $16 \mathrm{~V}$ and $625 \mathrm{~A}$, respectively. As a safety precaution for protecting the preheater from overheating, the preheater was provided with a temperature interlock. At the middle of the preheater, the wall temperature $T_{2}$ was measured and then fed to a high-temperature limit switch (Omega Engineering, Inc., Model CN76000) that would terminate power to the preheater when a preset upper-temperature limit was reached. After passing through the preheater, the test fluid entered the horizontal experimental test section. The experimental test section was heated, by passing current through its wall to generate resistance heat, with a direct current power supply (Electronic Measurements, Inc., Model EMHP 40-450-D-11111-0933). The output power could be regulated from 0 to $18 \mathrm{~kW}$ with the maximum voltage drop and the maximum current being $40 \mathrm{~V}$ and $450 \mathrm{~A}$, respectively. The voltage drop across the experimental test section $E$ was measured directly, and the current through the experimental test section $I$ was determined from a measurement of the voltage drop across a shunt resistor with known resistance of $0.0001 \Omega$. The heat input to the experimental test section was calculated using the product of the voltage drop and the current accounting for heat loss discussed subsequently. Electrical isolation for eliminating ground loops was provided for the preheater and the experimental test section by short high-pressure hoses, designated ISO in Fig. 1. The test fluid out from the experimental test section was cooled in the countercurrent heat exchanger that used laboratory water as a heat rejection fluid, and the cooled test fluid that left the heat exchanger returned to the pump to close the test loop.

The experimental test section was fabricated from a 2.9845-mm-inside-diameter and 4.7625mm-outside-diameter AISI type 316 stainless steel tube with a 0.9144-m heated length between 
the voltage taps. The in-stream bulk fluid temperatures were measured at the inlet and the outlet of the experimental test section with type $\mathrm{K}$ thermocouple probes (Omega Engineering, Inc.). A type K KMTSS-062U-6 thermocouple probe, whose very small outside diameter of $1.5748 \mathrm{~mm}$ allowed the test fluid passing through without significantly affecting the flow, was selected to measure the inlet bulk fluid temperature. Figure 1 also illustrates the method used to measure wall temperatures. The wall temperatures $T_{a}, T_{b}, \cdots, T_{j}$ were measured at 10 axial locations over the heated length of the experimental test section with type $\mathrm{K}$ thermocouple junctions (Omega Engineering, Inc.). To electrically isolate these thermocouple junctions from the experimental test section tube, a thin coat of high-temperature ceramic epoxy (Omega Engineering, Inc., Omega bond 200) was applied around the circumference of the tube at the measurement locations. After oven curing, the thermocouple junctions coated with the same high-temperature ceramic epoxy were bonded to the thin coating on the tube. This technique allowed the thermocouple junctions to be electrically insulated from the tube with current passing through it. The outlet fluid pressure $p_{\text {out }}$ and the overall pressure drop across the test section $\Delta p$ were measured in all tests with a piezoelectric pressure transducer (Endevco Corporation, Model 8510B-500) and a differential pressure transducer with variable reluctance (Validyne Engineering Corporation, Model DP15-36 1536N1S4A), respectively. These measurements were incorporated in the data reduction to calculate the friction factor across the experimental test section. As a safety precaution for protecting the experimental test section from overheating, the experimental test section was provided with a temperature interlock $T_{1}$ near the outlet of the experimental test section, and its wall temperature measurement was fed to a high-temperature limit switch (Omega Engineering, Inc., Model CN76000) that would terminate power to the experimental test section when a preset upper-temperature limit was reached. 
All the sensors for the measurements of the flowmeter temperature, the in-stream bulk fluid temperature, the wall temperature, the fluid pressure at outlet of the experimental test section, the overall pressure drop across the experimental test section, and the volumetric flowrate through the flowmeter were calibrated before installation. The flowmeter thermocouple probe, the instream temperature probes, and the wall thermocouple junctions were calibrated over the operation range with a type K reference probe (Omega Engineering Inc., No. 703998035), which was calibrated with the NIST-traceable standard. The pressure transducers were calibrated over the operation range using a precise pressure gauge (Ashcroft, Inc., Model Hiese CM-21615), which was calibrated with the NIST-traceable standard. The flowmeter was calibrated over the operation range using a weight-with-stopwatch technique. The correction equations developed based on the calibration data were incorporated into the data acquisition program.

\section{Heat loss calibration}

While the experimental test section was well insulated thermally from the atmosphere to minimize heat loss to the environment, heat loss tests were performed for the experimental test section wall temperatures up to the heat transfer test conditions. The heat loss was characterized through a special series of experiments with no fluid in the experimental test section. Power was applied to the experimental test section to bring its wall temperature to a selected level. The input power required for maintaining the wall temperature at the selected value is the heat loss rate $\dot{q}_{\text {loss }}$, which is related to the difference between the experimental test section wall temperature $T_{w}$ and the ambient temperature $T_{a m b}$. Therefore, by assuming a linear dependence on the

driving temperature, which was confirmed by the experimental results of the heat loss tests, the heat loss rate can be expressed approximately as 


$$
\dot{q}_{\text {loss }}=E I=c\left(T_{w}-T_{a m b}\right)
$$

where the proportional constant $c$, which depends on the heat transfer coefficient and the heat transfer surface area between the experimental test section and ambient for this particular experimental apparatus, was determined from the heat loss tests. Figure 2 shows the heat loss rate per length as a function of the driving temperature for the experimental test section. The test section heat loss was $<5 \%$ of the applied input power to the experimental test section in all subsequent heat transfer tests.

\section{Experimental data reduction}

The local convective heat transfer coefficient at position $z$ along the length of the test section is defined as

$$
h(z)=\frac{\dot{q}^{\prime \prime}(z)}{T_{w}(z)-T_{e}(z)}
$$

In the above equation, the local surface heat flux $\dot{q}^{\prime \prime}(z)$ was determined from the overall input heating power by using the local electrical resistivity as a function of the temperature along the experimental test section wall, with corrections for heat losses. The inner wall surface temperature of the experimental test section $T_{w}(z)$ was determined from a radial heat conduction calculation by using the measured outer surface temperature $T_{w}^{\prime}(z)$ and the local heat generated in the test section wall per unit length $\dot{q}^{\prime}(z)$

$$
T_{w}(z)=T_{w}^{\prime}(z)+\frac{\dot{q}^{\prime}(z)}{4 \pi k_{w}(z)} \frac{1+\ln \left(d_{i} / d_{o}\right)^{2}-\left(d_{i} / d_{o}\right)^{2}}{1-\left(d_{i} / d_{o}\right)^{2}}
$$


where $k_{w}(z)$ is the local thermal conductivity of the experimental test section wall as a function of the wall temperature, $d_{i}$ is the inside diameter of the experimental test section, and $d_{o}$ is the outside diameter of the experimental test section. The local nanofluid temperature $T_{e}(z)$ was obtained from a heat balance using the measured inlet nanofluid temperature and the test section heating power at the same location where the wall temperature $T_{w}(z)$ was measured.

In the above data reduction process and further analyses, nanofluid thermophysical properties including density, mass-specific heat capacity, dynamic viscosity, and thermal conductivity are necessary. In the present study, the effective density and the effective mass-specific heat capacity were calculated from the component properties and the particle volume concentration based on the physical principle of the mixture rule related to their definitions. For a nanofluid denoted by the subscript $e$ consisting of particles denoted by the subscript $p$ suspended in a base fluid denoted by the subscript $m$ with a mass of $m_{e}=m_{m}+m_{p}$ and a volume of $V_{e}=V_{m}+V_{p}$, the effective density $\rho_{e}$ is

$$
\rho_{e}=\frac{m_{e}}{V_{e}}=\frac{m_{m}+m_{p}}{V_{e}}=\frac{V_{m} \rho_{m}+V_{p} \rho_{p}}{V_{e}}=\left(1-v_{p}\right) \rho_{m}+v_{p} \rho_{p}
$$

where $v_{p}=V_{p} / V_{e}$ is the particle volume concentration. Similarly, for a thermal equilibrium nanofluid with a temperature change of $\Delta T=\Delta T_{m}=\Delta T_{p}=\Delta T_{e}$ under a heat input of $q_{e}=q_{m}+q_{p}$, the effective mass-specific heat capacity $c_{p e}$ is

$$
c_{p e}=\frac{q_{e}}{m_{e} \Delta T}=\frac{m_{m} c_{p m}+m_{p} c_{p p}}{m_{m}+m_{p}}=\frac{V_{m} \rho_{m} c_{p m}+V_{p} \rho_{p} c_{p p}}{V_{m} \rho_{m}+V_{p} \rho_{p}}=\frac{\left(1-v_{p}\right) \rho_{m} c_{p m}+v_{p} \rho_{p} c_{p p}}{\left(1-v_{p}\right) \rho_{m}+v_{p} \rho_{p}}
$$


The effective dynamic viscosities and the effective thermal conductivities of the nanofluids were experimentally measured. A rotational-type viscometer (Brookfield Engineering Laboratories, Model LVDV-II+P) was used to measure the dynamic viscosities of the nanofluids. Because the temperature usually plays an important role in the dynamic viscosity increases of nanofluids, the effective dynamic viscosity measurements were performed for temperatures up to $125{ }^{\circ} \mathrm{C}$ covering the temperature range of heat transfer experiments. Figure 3 shows the effective dynamic viscosities as a function of the temperature for the nanofluids with particle volume concentrations of $0.50 \%, 0.75 \%$, and $2.00 \%$, respectively, based on which fitting equations were developed. For the nanofluid with a particle volume concentration of $0.50 \%$, the effective viscosities are close to those of the base fluid at all temperatures. For nanofluids with higher particle volume concentrations, the effective viscosities are much higher at the ambient temperature, but decrease significantly with the temperature increase, approaching the base fluid viscosity at $125^{\circ} \mathrm{C}$. The effective thermal conductivities of the nanofluids were measured using a thermal conductivity probe (Decagon Devices, Inc., Model KD-2 Pro), which is based on the transient hot-wire method. The measurements of the effective thermal conductivities of the nanofluids were performed only at the ambient temperature due to accuracy limitations with nanofluids at elevated temperatures, and the measured effective thermal conductivity ratios of the nanofluids over the base fluid are 1.03, 1.10, and 1.19 for the nanofluids with particle volume concentrations of $0.50 \%, 0.75 \%$, and $2.00 \%$, respectively. As shown in Fig. 4, these enhancements are much higher than the predictions of the effective medium theory. Temperature independence of thermal conductivity enhancements in nanoparticle suspensions is predicted by the effective medium theory and previously observed in nanofluids that follow the effective medium theory predictions [15-17]. For nanofluids that show thermal conductivity 
enhancements above the effective medium theory predictions due to additional thermal conductivity enhancement mechanisms, significant thermal conductivity enhancements of nanofluids with the temperature were reported in the literature $[1,3,18]$. In the present study a conservative approach was adopted, and the same thermal conductivity enhancement ratios were assumed for higher temperatures although a degree of improvement is expected. These thermophysical property data were incorporated in the heat transfer data reduction.

\section{Base fluid heat transfer experiments}

To validate the experimental test apparatus and to establish baseline heat transfer data for comparison to nanofluid data, a series of heat transfer experiments with the base fluid Therminol 59 was carried out prior to nanofluid heat transfer experiments. The base fluid heat transfer

experiments were performed under similar conditions as the nanofluids. During the base fluid heat transfer experiments, the experimental parameters such as temperatures and flowrates were chosen to maintain turbulent flow conditions with the Reynolds numbers in the range of $4000<\operatorname{Re}<7000$. In Fig. 5, the Fanning friction factors calculated from the experimental pressure drop data were compared with the predicted values from the standard Blasius equation [19]

$$
f_{\text {Blasius }}=0.0791 \mathrm{Re}^{-0.25}
$$

As shown in Fig. 5, the experimental data are in reasonable agreement with the predicted values from the Blasius equation and all of the experimental data are within $\pm 20 \%$ of the predicted values, which is within the generally-accepted deviation range. In Fig. 6, the heat transfer 
coefficients were compared with the predicted values from the well-known Gnielinski equation [20]

$$
h=\frac{(f / 8)(\operatorname{Re}-1000) \operatorname{Pr}}{1+12.7(f / 8)^{1 / 2}\left(\operatorname{Pr}^{2 / 3}-1\right)}\left(k / d_{i}\right)
$$

where $\operatorname{Pr}$ is the Prandtl number, $k$ is the thermal conductivity, and $f$ is the friction factor calculated as

$$
f=(1.82 \log \operatorname{Re}-1.64)^{-2}
$$

It can be seen from Fig. 6 that the experimental data are in reasonable agreement with the predicted values from the Gnielinski equation and most of the experimental data are within $\pm 20 \%$ of the predicted values, which is within the generally-accepted deviation range.

\section{Nanofluid heat transfer results and discussion}

A series of experiments was carried out for a 0.50 vol. \% copper-in-Therminol 59 nanofluid and a 0.75 vol. \% copper-in-Therminol 59 nanofluid in turbulent flow with the flow velocity in the range of 3.0-5.0 m/s and the nanofluid temperature in the range of $70-90{ }^{\circ} \mathrm{C}$. In the following subsections, the heat transfer experimental results of the nanofluids are shown and discussed in detail.

\subsection{Heat transfer coefficient comparisons}

It has been shown that, for many nanofluids such as $\mathrm{Al}_{2} \mathrm{O}_{3}-\mathrm{in}-\mathrm{H}_{2} \mathrm{O}$ [21-13], $\mathrm{Cu}-\mathrm{in}-\mathrm{H}_{2} \mathrm{O}$ [24], CuO-in- $\mathrm{H}_{2} \mathrm{O}$ [20, 22], diamond-in- $\mathrm{H}_{2} \mathrm{O}$ [22, 26], multi-walled carbon nanotube-in- $\mathrm{H}_{2} \mathrm{O}$ [27], 
SiC-in- $\mathrm{H}_{2} \mathrm{O}$ [28, 29], $\mathrm{SiO}_{2}-\mathrm{in}_{2} \mathrm{H}_{2} \mathrm{O}$ [30], $\mathrm{TiO}_{2}-\mathrm{in}-\mathrm{H}_{2} \mathrm{O}$ [21], $\mathrm{TiO}_{2}-\mathrm{in}_{-} \mathrm{H}_{2} \mathrm{O}$ [31, 32], $\mathrm{ZrO}_{2}-\mathrm{in}_{-}-\mathrm{H}_{2} \mathrm{O}$ [22], and SiC-in-50/50 $\mathrm{C}_{2} \mathrm{H}_{6} \mathrm{O}_{2} / \mathrm{H}_{2} \mathrm{O}$ [33], a turbulent-flow nanofluid with a low particle volume concentration can normally be treated as a homogeneous fluid. Typically the nanoparticles are homogeneously dispersed in the base fluid, and therefore the heat transfer coefficient predictions from the standard single-phase equations will be very good if the effective thermophysical properties of the nanofluid are accurate [12]. In the present study, the base fluid Therminol 59 is synthetic oil with rather high viscosities especially for low temperatures, and the effective thermal conductivity enhancements of the copper-in-Therminol 59 nanofluids are beyond the predictions of the effective medium theory. Therminol 59 is outside of the data base range that produced the conclusion of the homogeneous treatment of nanofluids [12], and as such was treated independently. Therefore, as one of the purposes of the present study, the nanofluid heat transfer experimental data are compared with the predictions from the Blasius equation for the Fanning friction factor and the Gnielinski equation for the heat transfer coefficient under the turbulent-flow condition in Figs. 7-10. For the Fanning friction factor, as shown in Figs. 7 and 9, the experimental data generally are within $20 \%$ of the predictions from the Blasius equation with a relative mean deviation of $12.74 \%$ for the 0.50 vol. \% copper-in-Therminol 59 nanofluid and $8.70 \%$ for the 0.75 vol. \% copper-in-Therminol 59 nanofluid. For the heat transfer coefficient, as shown in Figs. 8 and 10, the experimental data generally are within 10\% of the predictions from the Gnielinski equation with a relative mean deviation of $8.00 \%$ for the 0.50 vol. \% copper-inTherminol 59 nanofluid and $5.66 \%$ for the 0.75 vol. \% copper-in-Therminol 59 nanofluid.

Another observation worth being pointed out is that, in addition to the thermophysical property effects, adding copper nanoparticles to the base fluid Therminol 59 causes further heat transfer enhancements. This phenomenon can be clearly seen by comparing Figs. 6, 8, and 10 
where the over-predictions of the Gnielinski equation over the nanofluid heat transfer coefficients reduce with the increase of the particle volume concentration with the highest overpredictions for the base fluid Therminol 59 and the lowest over-predictions for the 0.75 vol. $\%$ copper-in-Therminol 59 nanofluid. However, as will be shown in the subsequent discussion, the dominant influence factor on the heat transfer coefficient enhancements of the nanofluids over the base fluid Therminol 59 is their effective thermophysical properties. Both the good predictions of the Gnielinski equation for the nanofluid heat transfer coefficients and the dominant influence of the nanofluid thermophysical properties for the nanofluid heat transfer coefficient enhancements follow the trends shown in the engineering literature [12]. Thus, although Therminol 59 nanofluid properties are well outside the data base range that leads to the homogeneous treatment, the Therminol 59 nanofluid has now been shown to conform rather well to the resulting conclusions.

\subsection{Heat transfer coefficient enhancements}

Because the heat transfer experimental conditions such as the flow velocity and the fluid temperature usually are not exactly the same for nanofluid tests and for its base fluid tests, the general approach for analyzing the heat transfer performance of the nanofluid over its base fluid is to compare the experimentally-measured nanofluid heat transfer coefficients to the theoretically-predicted base fluid heat transfer coefficients. In the present study, while it predicts the base fluid experimental data reasonably well, the Gnielinski equation generally over-predicts the base fluid experimental heat transfer coefficients. To account for the difference and to better predict the base fluid experimental heat transfer coefficients of Fig. 6, the Reynolds number 
sensitivity was increased with a corresponding change in the leading constant in the DittusBoelter equation [34] resulting in the following correlation

$$
h=0.002515 \operatorname{Re}^{1.015} \operatorname{Pr}^{0.4}\left(k / d_{i}\right)
$$

As shown in Fig. 11, the base fluid experimental heat transfer coefficients are in very good agreement with the predictions from the fitting equation, and all of the base fluid experimental data are within $\pm 5 \%$ of the predictions with a relative mean deviation of $2.85 \%$.

The heat transfer coefficient ratios of the experimental nanofluid data over the predicted base fluid values from Eq. (9) are plotted as a function of the flow velocity in Fig. 12 for the 0.50 vol. \% copper-in-Therminol 59 nanofluid and Fig. 13 for the 0.75 vol. \% copper-in-Therminol 59 nanofluid. Also shown in Figs. 12 and 13 are the predicted heat transfer coefficient ratios $h_{e} / h_{m}$, which is equivalent to the Mouromtseff number ratios $\mathrm{Mo}_{e} / \mathrm{Mo}_{m}$ [35] on the constant flow velocity comparison basis $[12,36]$ and, for a turbulent flow with the heat transfer coefficient predicted by the Dittus-Boelter equation [34], can be expressed as

$$
\frac{h_{e}}{h_{m}}=\frac{\mathrm{Mo}_{e}}{\mathrm{Mo}_{m}}=\left(\frac{\rho_{e}}{\rho_{m}}\right)^{0.8}\left(\frac{c_{p e}}{c_{p m}}\right)^{0.4}\left(\frac{\mu_{e}}{\mu_{m}}\right)^{-0.4}\left(\frac{k_{e}}{k_{m}}\right)^{0.6}
$$

where $\mu$ is the dynamic viscosity. It can be seen from Figs. 12 and 13 that (a) the heat transfer coefficient ratios of the nanofluids over the base fluid are quite uniform over the flow velocity range tested in the present study and (b) the heat transfer coefficient ratios of the experimental nanofluid data over the predicted base fluid values are in very good agreement with the predicted Mouromtseff number ratios based on the thermophysical properties of the nanofluids and the base fluid Therminol 59. The relative mean deviation is $2.18 \%$ for the 0.50 vol. $\%$ copper-in- 
Therminol 59 nanofluid and $2.83 \%$ for the 0.75 vol. \% copper-in-Therminol 59 nanofluid. These results follow the trends shown in the engineering literature [12].

\subsection{Temperature effects on heat transfer coefficient enhancements}

Based on the above results and discussion, it is very reasonable to assume that the heat transfer coefficient ratios of the copper-in-Therminol 59 nanofluids over the base fluid Therminol 59 can generally be predicted from their thermophysical properties for temperatures beyond the heat transfer experimental range of this study. In the present study, the temperature effects on the nanofluid density and the nanofluid mass-specific heat capacity are implied in Eqs. (4) and (5). The nanofluid thermal conductivity enhancements are assumed to be unchanged with the temperature. The temperature effects on the nanofluid dynamic viscosities are reflected in the fitting equations for temperatures within the experimental viscosity measurement range. For temperatures higher than the experimental viscosity measurement range, because the nanofluid dynamic viscosities quickly approach the base fluid dynamic viscosities as shown in Fig. 3, it is reasonable to assume that they follow the Einstein equation [37] (which is generally the case at elevated temperatures and low particle volume concentrations)

$$
\mu_{e}=\left(1+2.5 v_{p}\right) \mu_{m}
$$

This presumption is used in the present study with temperatures higher than $200{ }^{\circ} \mathrm{C}$ up to the highest Therminol 59 operating temperature of $315^{\circ} \mathrm{C}$ for the copper-in-Therminol 59 nanofluids with particle volume concentrations of $0.50 \%, 0.75 \%$, and $2.00 \%$, respectively.

Under those presumptions, the heat transfer coefficient ratios or equivalently the Mouromtseff number ratios calculated from Eq. (10) for the copper-in-Therminol 59 nanofluids 
at various particle volume concentrations of $0.50 \%, 0.75 \%$, and $2.00 \%$ in turbulent flow with the heat transfer coefficients predicted by the Dittus-Boelter equation are plotted as a function of the temperature in Fig. 14. It can be seen from Fig. 14 that the average heat transfer coefficient ratios of the nanofluid over the base fluid Therminol 59 are approximately 1.04, 1.09, and 1.18, respectively for the copper particle volume concentrations of $0.50 \%, 0.75 \%$, and $2.00 \%$ respectively. These results are higher than those obtained from the experiments in the lower temperature range, which reflects the temperature effects on one of the important thermophysical properties affecting the heat transfer coefficients, the nanofluid dynamic viscosity. These levels of heat transfer enhancements at elevated temperatures are very attractive for the common hightemperature heat transfer applications of Therminol 59.

\section{Conclusions}

Heat transfer experiments were carried out for the particular copper-in-Therminol 59 nanofluids of which base fluid thermal conductivities decrease with the temperature increase, base fluid viscosities are rather high, especially at low temperatures, and effective thermal conductivity enhancements are beyond the predictions of the effective medium theory. The former is a negative and the latter a positive effect on the heat transfer performance of the nanofluids. The experimental data show that (a) the measured Fanning friction factors and heat transfer coefficients of the copper-in-Therminol 59 nanofluids at particle volume concentrations of $0.50 \%$ and $0.75 \%$ are well predicted by the Blasius equation and the Gnielinski equation; (b) while there are additional effects of copper nanoparticles on the nanofluid convective turbulentflow heat transfer coefficients, they are insignificant compared to the dominant influence factor, the effective thermophysical properties of the nanofluids; and (c) the experimental heat transfer 
coefficient enhancements are well predicted by the heat transfer coefficient ratios of the nanofluids over the base fluid using the Dittus-Boelter equation for turbulent-flow heat transfer coefficient calculations based on the thermophysical properties of the nanofluids and the base fluid. Based on the above results from this study, it is shown that, for high temperatures, the heat transfer coefficients of the copper-in-Therminol 59 nanofluids, calculated from the predicted thermophysical properties of the nanofluids, are improved as much as $18 \%$ by the introduction of low concentrations $(<2.00$ vol. \%) of copper nanoparticles. Because Therminol 59 is a commonly-used high-temperature heat transfer fluid, these results make copper-in-Therminol 59 nanofluids very attractive for many commercial applications.

\section{Acknowledgements}

This work was sponsored by the US Department of Energy's Solar Energy Technology Program Office, American Recovery and Reinvestment Act (ARRA) funding, under contract number DE-AC02-06CH11357 at Argonne National Laboratory, managed by UChicago Argonne LLC. Experimental assistance by J. Gaviria and K. Velvadapu is acknowledged.

\section{References}

[1] S.K. Das, S.U.S. Choi, H.E. Patel, Heat transfer in nanofluids - a review, Heat Transfer Engineering 27 (10) (2006) 3-19.

[2] X.-Q. Wang, A. S. Mujumdar, Heat transfer characteristics of nanofluids: a review, International Journal of Thermal Sciences 46 (2007) 1-19. 
[3] W. Yu, D.M. France, S.U.S. Choi, J.L. Routbort, Review and comparison of nanofluid thermal conductivity and heat transfer enhancements, Heat Transfer Engineering 29 (2008) $432-460$.

[4] Y. Li, J. Zhou, S. Tung, E. Schneider, S. Xi, A review on development of nanofluid preparation and characterization, Powder Technology 196 (2009) 89-101.

[5] D. Wu, H. Zhu, L. Wang, L. Liu, Critical issues in nanofluids preparation, characterization and thermal conductivity, Current Nanoscience 5 (2009) 103-112.

[6] V.I. Terekhov, S.V. Kalinina, V.V. Lemanov, The mechanism of heat transfer in nanofluids: state of the art (review), part 1. synthesis and properties of nanofluids, Thermophysics and Aeromechanics 17 (2010) 1-15.

[7] S. Özerinç, S. Kakaç, A.G. Yazicioğlu, Enhanced thermal conductivity of nanofluids: a state-of-the-art review, Microfluidics and Nanofluidics 8 (2010) 145-170.

[8] J.C. Maxwell, A Treatise on Electricity and Magnetism, Clarendon Press, Oxford, England, 1873.

[9] D.A.G. Bruggeman, Berechnung verschiedener physikalisher Konstanten von heterogenen Substanzen: I. Dielektrizitätskonstanten und Leitfähigkeiten der Mischkörper aus isotropen Substanzen, Annalen der Physik 24 (1935) 636-664.

[10] C. Okhio, D. Hodges, J. Black, Review of literature on nanofluid flow and heat transfer properties, Cyber Journals: Multidisciplinary Journals in Science and Technology, Journal of Selected Areas in Nanotechnology (JSAN) 1 (2010) 1-8.

[11] A. Einstein, Eine neue Bestimmung der Moleküldimensionen, Annalen der Physik 19 (1906) 289-306. 
[12] W. Yu, D.M. France, E.V. Timofeeva, D. Singh, J.L. Routbort, Comparative review of turbulent heat transfer of nanofluids, International Journal of Heat and Mass Transfer 55 (2012) 5380-5396.

[13] S.A. Kumar, K.S. Meenakshi, B.R.V. Narashimhan, S. Srikanth, G. Arthanareeswaran, Synthesis and characterization of copper nanofluid by a novel one-step method, Materials Chemistry and Physics 113 (2009) 57-62.

[14] X.M. Lin, C.M. Sorensen, K.J. Klabunde, Digestive ripening, nanophase segregation and superlattice formation in gold nanocrystal colloids, Journal of Nanoparticle Research 2 (2000) 157-164.

[15] E.V. Timofeeva, A.N. Gavrilov, J.M. McCloskey, Y.V. Tolmachev, S. Sprunt, L.M. Lopatina, J.V. Selinger, Thermal conductivity and particle agglomeration in alumina nanofluids: experiment and theory, Physical Review E 76 (2007) $061203-061216$.

[16] D. Singh, E. Timofeeva, W. Yu, J. Routbort, D. France, D. Smith, J.M. Lopez-Cepero, An investigation of silicon carbide-water nanofluid for heat transfer applications, Journal of Applied Physics 105 (2009) 064306.

[17] A. Ehle, S. Feja, M. H. Buschmann, Temperature dependency of ceramic nanofluids shows classical behaviour, Journal of Thermophysics and Heat Transfer 25 (2011) 378-385.

[18] S.M.S. Murshed, K.C. Leong, C. Yang, Thermophysical and electrokinetic properties of nanofluids - a critical review, Applied Thermal Engineering 28 (2008) 2109-2125.

[19] H. Blasius, Das Ähnlichkeitsgesetz bei Reibungsvorgängen in Flüssigkeiten, Forschungsarbeiten des Ingenieurwesens, Heft 131, 41 pp., Verein Deutscher Ingenieure, Berlin, 1913. 
[20] Von V. Gnielinski, Neue Gleichungen für den Wärme- und den Stoffübergang in turbulent durchströmten Rohren und Kanälen, Forschung im Ingenieurwesen 41 (1975) 8-16.

[21] B.C. Pak, Y.I. Cho, Hydrodynamic and heat transfer study of dispersed fluids with submicron metallic oxide particles, Experimental Heat Transfer 11 (1998) 151-170.

[22] W. Williams, J. Buongiorno, L.-W. Hu, Experimental investigation of convective heat transfer and pressure loss of alumina/water and zirconia/water nanoparticle colloids (nanofluids) in horizontal tubes, Transactions of the ASME, Journal of Heat Transfer 130 (2008) 042412.

[23] S. Torii, Turbulent heat transfer behavior of nanofluid in a circular tube heated under constant heat flux, Advances in Mechanical Engineering (2010) 917612.

[24] Y. Xuan, Q. Li, Investigation on convective heat transfer and flow features on nanofluids, Transactions of the ASME, Journal of Heat Transfer 125 (2003) 151-155.

[25] S.M. Fotukian, M. Nasr Esfahany, Experimental study turbulent convective heat transfer and pressure drop of dilute $\mathrm{CuO} /$ water nanofluid inside a circular tube, International Communications in Heat and Mass Transfer 37 (2010) 214-219.

[26] S.M. Fotukian, M. Nasr Esfahany, Experimental study turbulent convective heat transfer and pressure drop of dilute $\mathrm{CuO} /$ water nanofluid inside a circular tube, International Communications in Heat and Mass Transfer 37 (2010) 214-219.

[27] L. Liao, Z.-H. Liu, Forced convective flow drag and heat transfer characteristics of carbon nanotube suspensions in a horizontal small tube, Heat and Mass Transfer 45 (2009) 11291136. 
[28] W. Yu, D.M. France, D. S. Smith, D. Singh, E.V. Timofeeva, J.L. Routbort, Heat transfer to a silicon carbide/water nanofluid, International Journal of Heat and Mass Transfer 52 (2009) 3606-3612.

[29] E.V. Timofeeva, D.D. Smith, W. Yu, D.M. France, D. Singh, J.L. Routbort, Particle size and interfacial effects on heat transfer characteristics of water and $\alpha$-SiC nanofluids, Nanotechnology 21 (2010) 215703.

[30] S. Ferrouillat, A. Bontemps, J.-P. Ribeiro, J.-A. Gruss, O. Soriano, Hydraulic and heat transfer study of $\mathrm{SiO}_{2}$ /water nanofluids in horizontal tubes with imposed wall temperature boundary conditions, International Journal of Heat and Fluid Flow 32 (2011) 424-439.

[31] W. Duangthongsuk, S. Wongwises, Heat transfer enhancement and pressure drop characteristics of $\mathrm{TiO}_{2}$-water nanofluid in a double-tube counter flow heat exchanger, International Journal of Heat and Mass Transfer 52 (2009) 2059-2067.

[32] W. Duangthongsuk, S. Wongwises, An experimental study on the heat transfer performance and pressure drop of $\mathrm{TiO}_{2}$-water nanofluids flowing under a turbulent flow regime, International Journal of Heat and Mass Transfer 53 (2010) 334-344.

[33] E.V. Timofeeva, W. Yu, D.M. France, D. Singh, J.L. Routbort, Base fluid and temperature effects on the heat transfer characteristics of $\mathrm{SiC}$ in $\mathrm{EG} / \mathrm{H}_{2} \mathrm{O}$ and $\mathrm{H}_{2} \mathrm{O}$ nanofluids, Journal of Applied Physics 109 (2011) 014914.

[34] F.W. Dittus and L.M.K. Boelter, Heat transfer in automobile radiators of the tubular type, University of California Publications in Engineering 2 (1930) 443-461.

[35] I.E. Mouromtseff, Water and forced-air cooling of vacuum tubes, Proceedings of the IRE 30 (1942) 190-205. 
[36] W. Yu, D.M. France, E.V. Timofeeva, D. Singh, J.L. Routbort, Thermophysical propertyrelated comparison criteria for nanofluid heat transfer enhancement in turbulent flow, Applied Physics Letters 96 (2010) 213109.

[37] A. Einstein, Eine neue Bestimmung der Moleküldimensionen, Annalen der Physik 19 (1906) 289-306.

\section{Figure Captions}

Fig. 1. Schematic diagram of experimental apparatus.

Fig. 2. Heat loss calibration.

Fig. 3. Nanofluid viscosities as a function of the temperature.

Fig. 4. Nanofluid thermal conductivities as a function of the particle volume concentration.

Fig. 5. Base fluid Fanning friction factor comparison.

Fig. 6. Base fluid heat transfer coefficient comparison.

Fig. 7. 0.50 vol. \% Cu-in-Therminol 59 nanofluid Fanning friction factor comparison.

Fig. 8. 0.50 vol. \% Cu-in-Therminol 59 nanofluid heat transfer coefficient comparison.

Fig. 9. 0.75 vol. \% Cu-in-Therminol 59 nanofluid Fanning friction factor comparison.

Fig. 10. 0.75 vol. \% Cu-in-Therminol 59 nanofluid heat transfer coefficient comparison.

Fig. 11. Base fluid heat transfer coefficient correlation.

Fig. 12. 0.50 vol. \% Cu-in-Therminol 59 nanofluid heat transfer coefficient enhancement.

Fig. 13. 0.75 vol. \% Cu-in-Therminol 59 nanofluid heat transfer coefficient enhancement.

Fig. 14. Temperature effect on nanofluid heat transfer coefficient enhancement. 


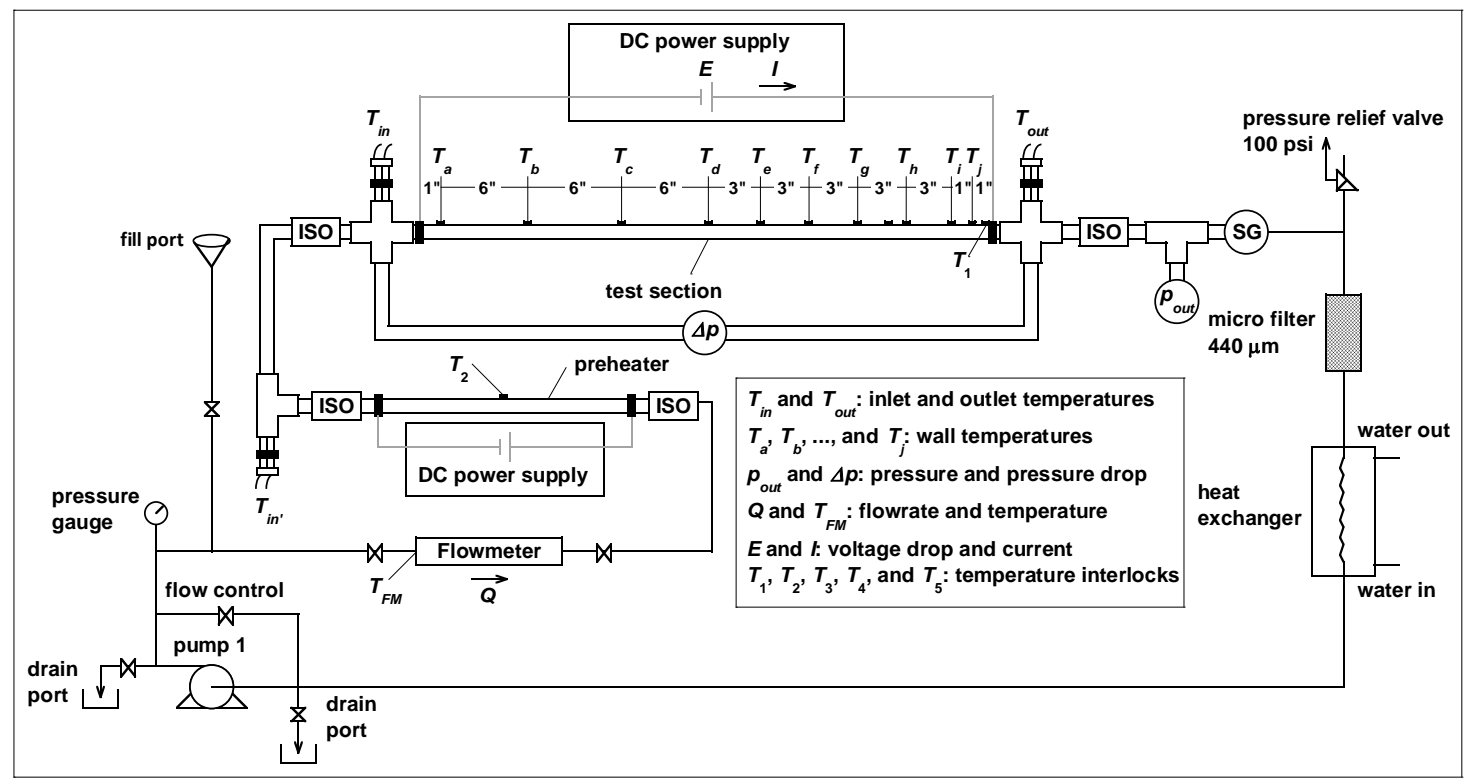

Fig. 1. Schematic diagram of experimental apparatus. 


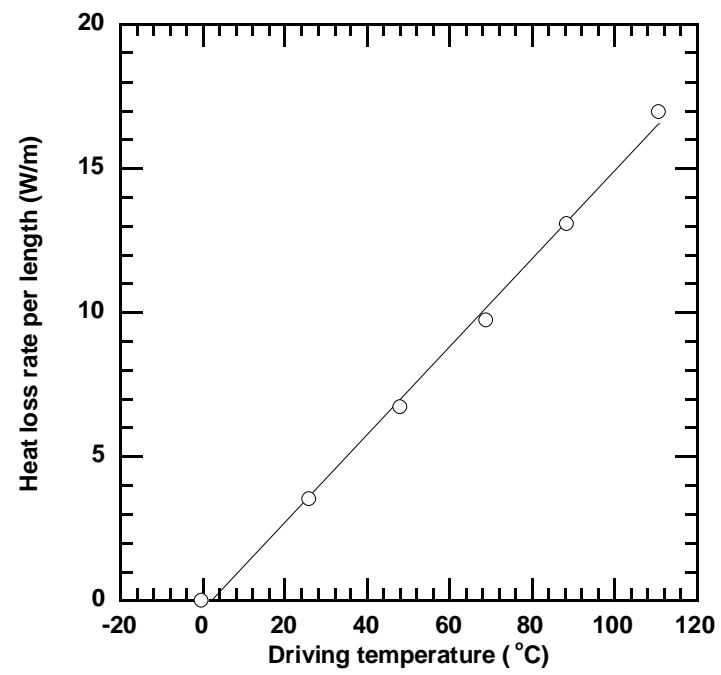

Fig. 2. Heat loss calibration. 


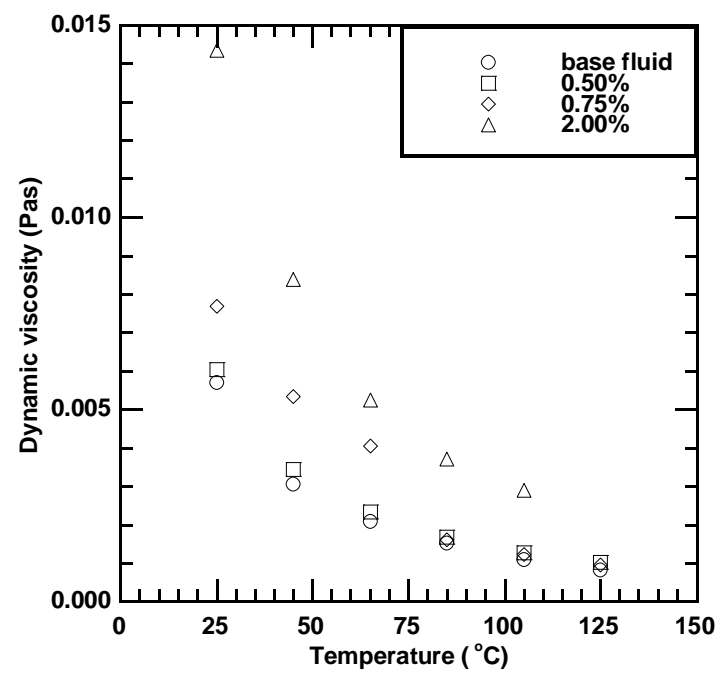

Fig. 3. Nanofluid viscosities as a function of the temperature. 


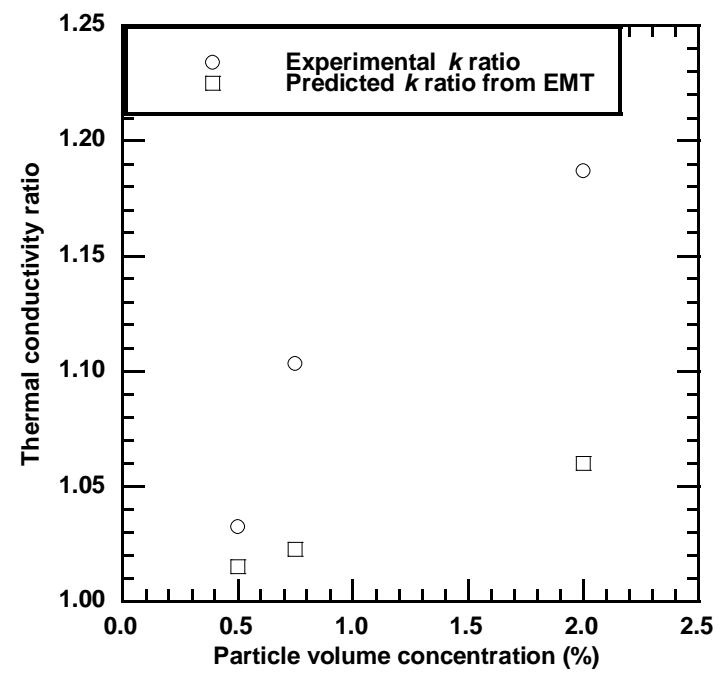

Fig. 4. Nanofluid thermal conductivities as a function of the particle volume concentration. 


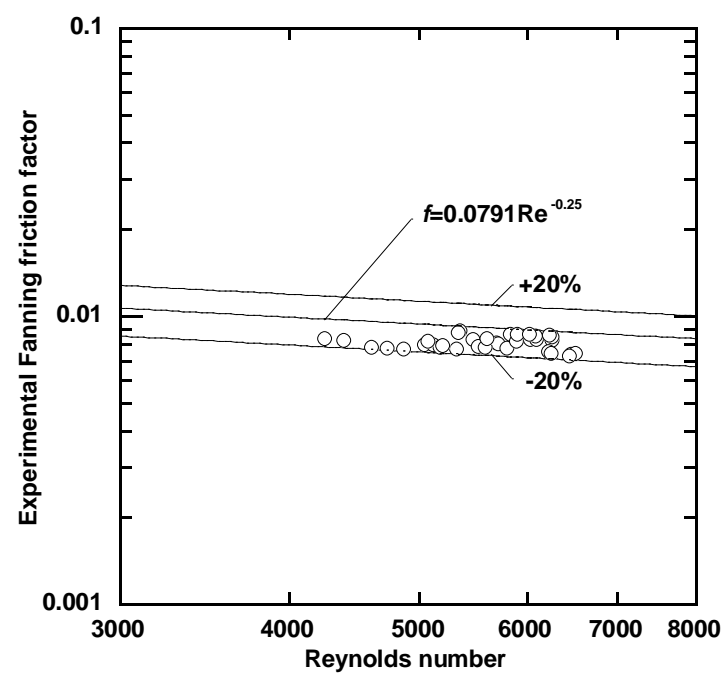

Fig. 5. Base fluid Fanning friction factor comparison. 


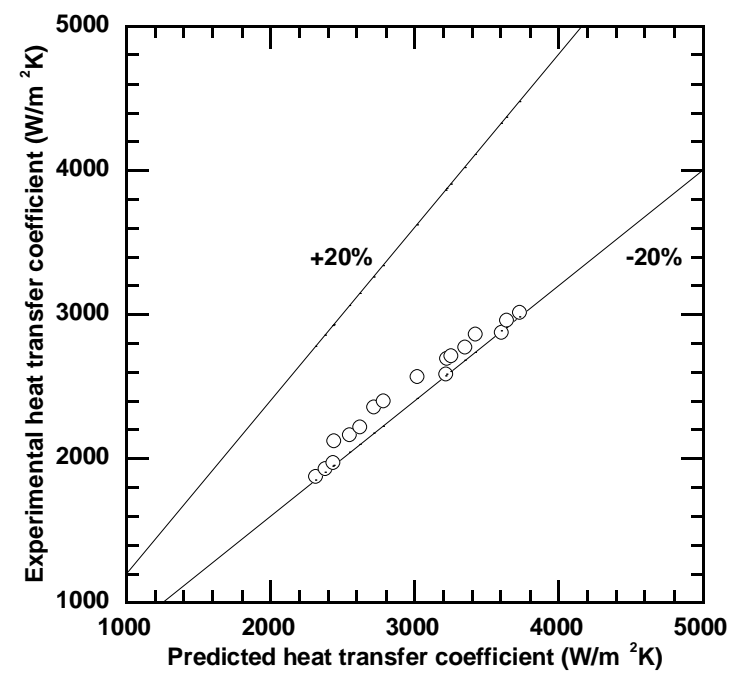

Fig. 6. Base fluid heat transfer coefficient comparison. 


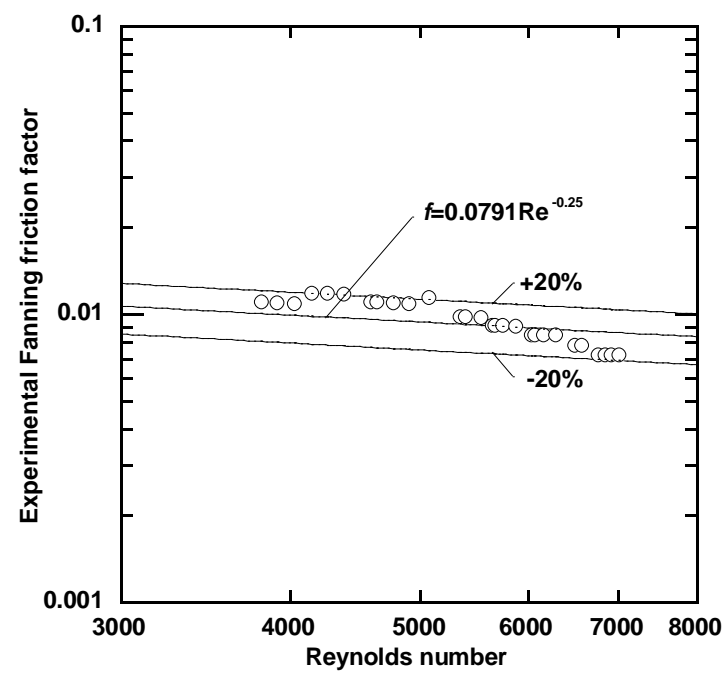

Fig. 7. 0.50 vol. \% Cu-in-Therminol 59 nanofluid Fanning friction factor comparison. 


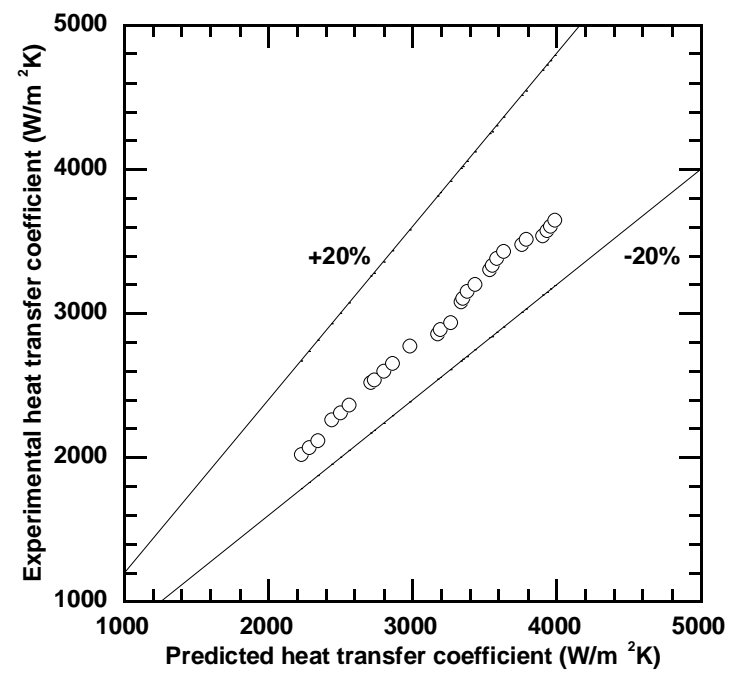

Fig. 8. 0.50 vol. \% Cu-in-Therminol 59 nanofluid heat transfer coefficient comparison. 


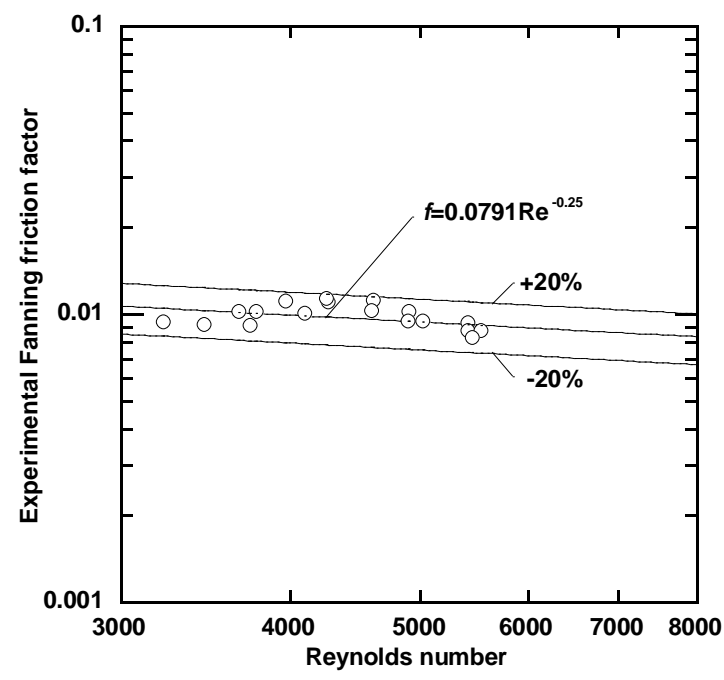

Fig. 9. 0.75 vol. \% Cu-in-Therminol 59 nanofluid Fanning friction factor comparison. 


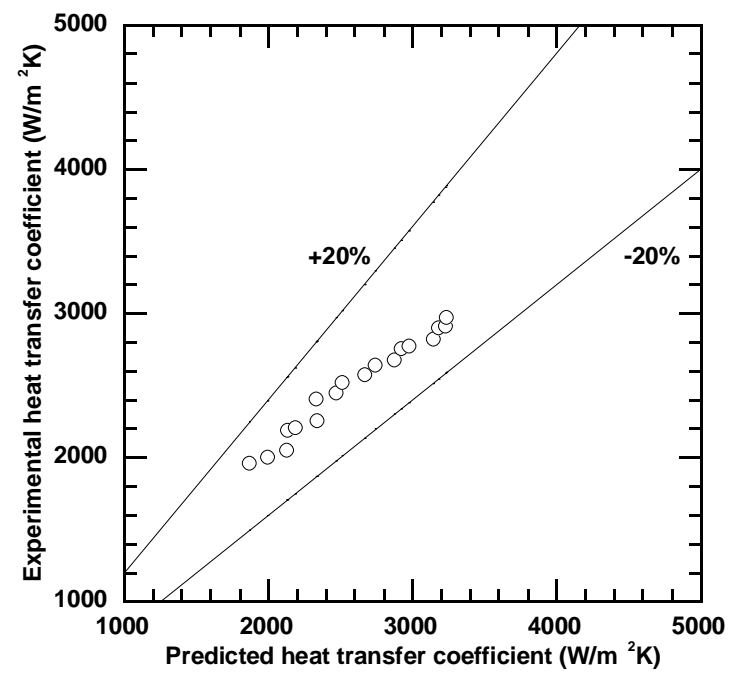

Fig. 10. 0.75 vol. \% Cu-in-Therminol 59 nanofluid heat transfer coefficient comparison. 


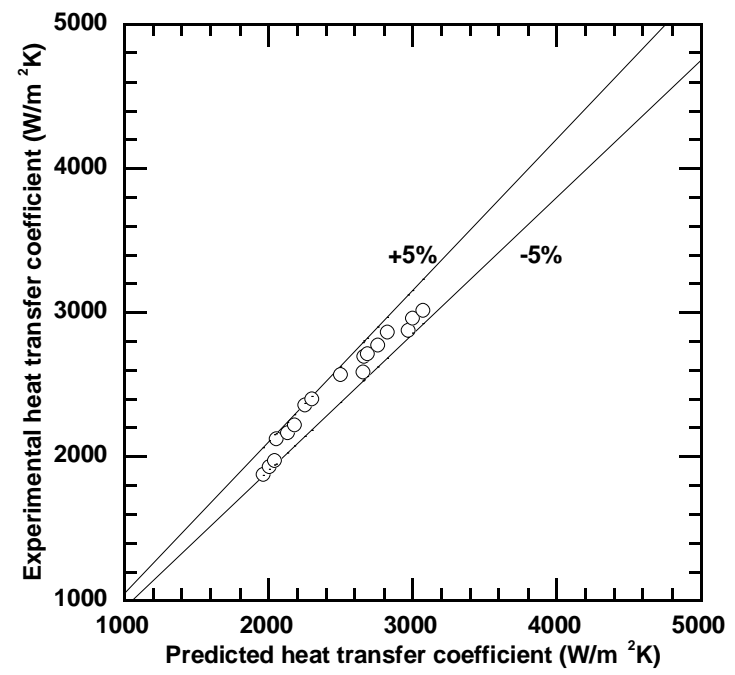

Fig. 11. Base fluid heat transfer coefficient correlation. 


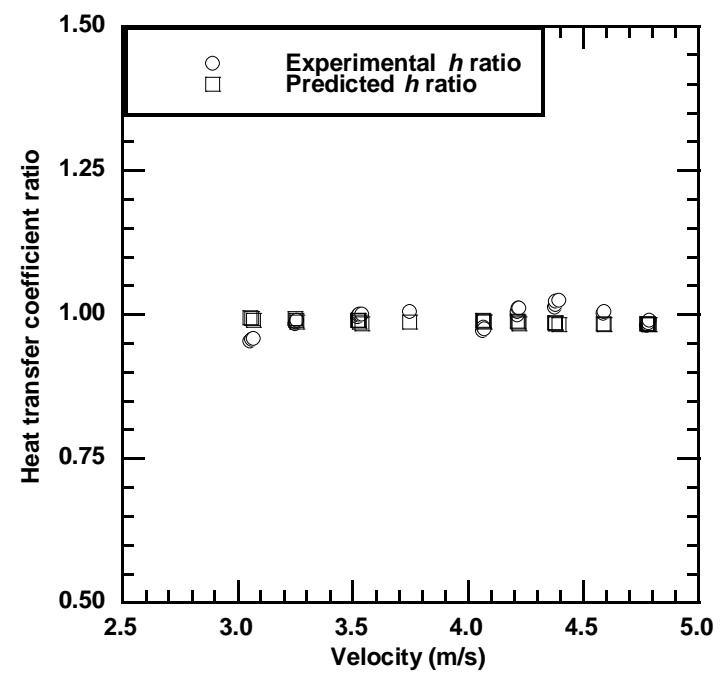

Fig. 12. 0.50 vol. \% $\mathrm{Cu}$-in-Therminol 59 nanofluid heat transfer coefficient enhancement. 


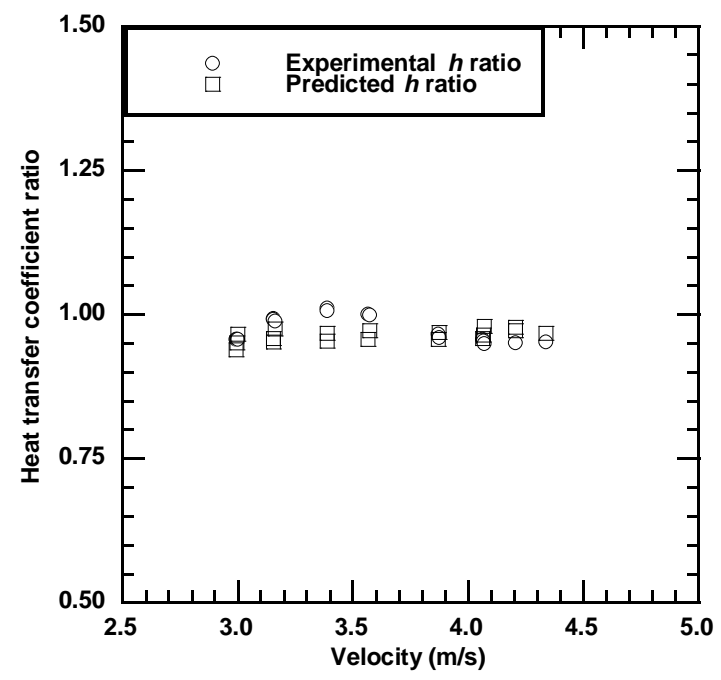

Fig. 13. 0.75 vol. \% $\mathrm{Cu}$-in-Therminol 59 nanofluid heat transfer coefficient enhancement. 


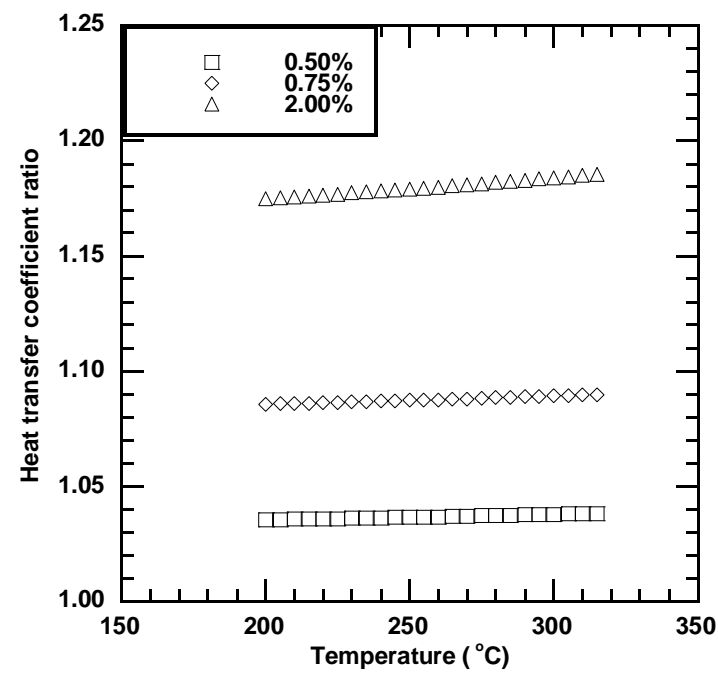

Fig. 14. Temperature effect on nanofluid heat transfer coefficient enhancement. 